\title{
El periódico como campo de tensiones: un análisis del despliegue informativo y publicitario
}

\author{
Esteban Andrés Zunino \\ Consejo Nacional de Investigaciones Científicas y Técnicas (CONICET) \\ Universidad Nacional de Quilmes \\ estebanzunino@hotmail.com
}

\section{Resumen}

Recibido: 22 de junio de 2016.

El objetivo general del presente trabajo es analizar y operacionalizar el concepto de hueco informativo a partir del estudio de la cobertura mediática del conflicto entre el gobierno argentino y las principales corporaciones agrarias del país en 2008. Se desplegó un análisis de contenido cuantitativo sobre los 384 ejemplares publicados por los diarios Clarín, La Nación y Página/12 durante los 128 días de contienda. Los resultados del trabajo empírico demostraron, además de una alta relevancia del caso en la agenda mediática, la existencia de una fuerte competencia entre los contenidos informativos y publicitarios. En ese sentido, se sostiene que el hueco informativo no debe ser considerado como un compartimento estanco, puesto que es habitualmente condicionado por el contenido publicitario, factor central del financiamiento de las empresas periodísticas.

Palabras clave: hueco informativo, periódicos, conflicto agrario, publicidad, conflictos sociales, agenda setting, Argentina.

\section{The newspaper as a contested field: analyzing the distribution of in- formation and advertisements}

\begin{abstract}
The main objective of this paper is to analyze and operationalize the concept of the newshole by studying media coverage of the conflict between the Argentine government and the nation's main agricultural corporations in 2008. We have carried out a quantitative content analysis of the 384 issues published by the newspapers Clarín, La Nación, and Página/12 during the 128 days of the conflict. The results of this empirical study have demonstrated that, beyond the importance of this case in the media agenda, there was also a high degree of competition between informative and advertising contents. In this sense, we maintain that the newshole should not be thought of as an airtight container, since it is habitually conditioned by advertising content, which plays a crucial role in the financing of newspapers.
\end{abstract}

Keywords: newshole, newspapers, agricultural conflict, advertising, social conflicts, agenda setting, Argentina. 


\title{
O jornal como campo de tensões: una análise do desdobramento in- formativo e publicitário
}

\begin{abstract}
Resumo
O objetivo geral do presente trabalho é analisar e operacionalizar o conceito de buraco informativo a partir do estudo da cobertura midiática do conflito entre o governo argentino e as principais corporações agrárias do país no ano 2008. Desenvolveu-se uma análise de conteúdo quantitativo sobre os 384 exemplares publicados pelos jornais Clarín, La Nación e Página/12 durante os 128 dias de disputa. Os resultados do trabalho empírico demostraram, além de uma alta relevância do caso na agenda midiática, a existência de uma forte competência entre os conteúdos informativos e publicitários. Nesse sentido, se sustenta que o buraco informativo não deve ser considerado como um compartimento estanque, já que é habitualmente condicionado pelo conteúdo publicitário, fator central do financiamento das empresas jornalísticas.
\end{abstract}

Palavras chave: buraco informativo, jornais, conflito agrário, publicidade, conflitos sociais, agenda setting, Argentina.

\section{Introducción}

El concepto de hueco informativo (news hole) remite a la totalidad del espacio que los periódicos dedican a la información una vez descontada la publicidad (Budd, 1964). Tanto en diarios como en programas audiovisuales e incluso en medios digitales, el concepto remite a la noción de que el tamaño de las agendas informativas es siempre limitado (McCombs, 2006).

Lo acotado del news hole genera una competencia temática entre los diferentes asuntos por ocupar sitios destacados en la agenda. Tal competencia se da en un juego de suma cero, por lo que el aumento de la frecuencia de cobertura sobre un caso y/o el incremento de su jerarquía acotan la posibilidad de que otros temas ingresen o sean visibles en la superficie mediática (Zhu, 1992).

Aun cuando las nuevas tecnologías han ampliado el volumen de inclusión y almacenamiento de noticias, este colisiona con las severas restricciones que impone al desarrollo informativo la capacidad también limitada del público de recordar de manera vívida no más que un puñado de temas. En efecto, diversos autores consideran que la audiencia no es capaz de recordar más de cuatro o cinco temas luego de haberse expuesto a los mensajes mediáticos (Zhu, 1992; McCombs, 2006; Brosius \& Kepplinger, 1992).

El objetivo general de esta investigación es sistematizar y operacionalizar el concepto de hueco informativo a partir del estudio de la cobertura mediática del conflicto desatado entre las principales corporaciones agropecuarias de la Argentina y el gobierno de la presidente Cristina Fernández de Kirchner luego de la decisión del Poder Ejecutivo de modificar el cuadro de gravámenes a las principales producciones del sector en 2008. Para ello se desarrolló un análisis de contenido cuantitativo sobre todos 
los ejemplares y las piezas informativas publicadas al respecto durante 128 días por tres diarios de importancia nacional: Clarín, La Nación y Página/12.

En términos específicos se pretende 1) establecer qué tipo de relación existe entre la relevancia de un caso y el espacio total disponible para la información; 2) determinar si el aumento de información sobre un tema de alto impacto económico, político y social genera modificaciones en la cantidad total de espacio disponible para las noticias en la agenda mediática; y 3) vislumbrar qué tipo de factores externos al hueco informativo ejercen presión sobre la disposición de las noticias en la superficie mediática.

\section{El caso bajo estudio}

El 11 de marzo de 2008, un día después de su publicación en el Boletín oficial, el gobierno argentino anunció la Resolución No 125/08 del Ministerio de Economía ${ }^{1}$ que elevaba los gravámenes que debían tributar los principales commodities que produce el sector agropecuario.

El nuevo cuadro subía los impuestos a la exportación de soja del 35\% al 44,1\% y de girasol del 32,1\% al 39,1\%, mientras que se reducían las alícuotas para el maíz y el trigo en un $1 \%$. La nueva modalidad establecía una variación en la carga impositiva, asociada a la cotización de los granos en el mercado internacional. El gobierno de la presidente Cristina Fernández justificó la implementación de estas medidas con el argumento de que servirían para desacoplar los precios de las materias primas para el mercado local de las cotizaciones internacionales (Barsky \& Gelman, 2009; Novaro, Bonvechi \& Cherny, 2014).

El titular de la Ministerio de Hacienda realizó estos anuncios días antes del comienzo de la época de cosecha, por lo que productores rurales grandes, medianos y pequeños, agrupados en la Sociedad Rural Argentina (SRA), la Federación Agraria Argentina (FAA), las Confederaciones Rurales Argentinas (CRA) y la Confederación Intercooperativa Agropecuaria Cooperativa Limitada (CONINAGRO) se agruparon en un organismo centralizado denominado Comisión de Enlace, desde el cual llevaron a cabo medidas de protesta como cortes de ruta y cese de comercialización de granos.

El conflicto se constituyó en un hecho central de las agendas mediática y pública durante 128 días (Amado \& Rotelli, 2011; Becerra \& López, 2009; Cremonte, 2010). Transcurridos más de tres meses de pugna, el Poder Ejecutivo Nacional (PEN) decidió enviar la norma al Congreso Nacional para su discusión. Finalmente, la Resolución $\mathrm{N}^{\circ}$ 125/08 fue derogada por el Senado, entradas las primeras horas del día jueves 17 de

\footnotetext{
1 El texto completo de la Resolución № 125/08 puede obtenerse en: http://servicios.infoleg.gob.ar/infolegInternet/ anexos/135000-139999/138567/texact.htm
} 
julio de 2008. El exvicepresidente de la Nación y presidente de la Cámara de Senadores, Julio Cobos, definió la votación -que hasta ese momento estaba empatada en 36 votos- con la frase "Mi voto es no positivo".

\section{Marco teórico: hueco informativo y suma cero}

El concepto de agenda mediática se inscribe en la perspectiva teórica de la agenda setting. Esta teoría sostiene la existencia de un efecto causal, acumulativo y de carácter cognitivo de los medios de comunicación sobre la audiencia (McCombs \& Shaw, 1972). De acuerdo con esta hipótesis, los medios son capaces de afectar la percepción del público en dos niveles diferentes: el de la relevancia y el de los atributos (Weaver, McCombs \& Shaw, 2004). El primero de ellos remite a la existencia de una correlación significativa entre la importancia que los medios les asignan a los temas - a partir de su reiteración y ubicación en sitios destacados-y la que los sujetos les conceden a esos mismos asuntos. El segundo refiere a ciertos rasgos afectivos y cognitivos presentes en las noticias que suelen moldear la percepción del público sobre los objetos (Aruguete, 2015).

Los temas que componen la agenda mediática son definidos como "la acumulación de una serie de acontecimientos relacionados que se involucran en el tratamiento periodístico y que se agrupan unidos en una categoría más amplia" (Shaw, 1977, citado en Dader, 1992, p. 302). La construcción de la agenda mediática implica en el seno de las redacciones una secuencia de procesos de selección temática y jerarquización de las noticias a partir de los cuales los medios de comunicación completan su hueco informativo, definido como la cantidad del espacio disponible para el material no publicitario (McCombs, 2006). Dada su espacialidad acotada, los diferentes issues compiten por obtener un lugar en él en un juego en el que la entrada de un nuevo tema afecta la permanencia de los restantes (Budd, 1964; McCombs, 1992).

Los límites en el tamaño de la mayoría de las agendas mediáticas resultan más obvios, al darse una cantidad limitada de espacio en el periódico y una cantidad limitada de tiempo en las radiodifusiones informativas. Incluso en el caso de los sitios web de internet, con su capacidad aparentemente ilimitada de ir añadiendo páginas, nos encontramos con que el alcance y el tiempo disponible de la atención pública imponen severas restricciones (McCombs, 2006, p. 85).

En efecto, se sostiene que la competencia temática y, por lo tanto, el proceso de agenda setting, constituye un juego de suma cero, ya que existen "por un lado, el vasto número de temas sociales que se resaltan y, por el otro, la capacidad limitada que la agenda pública tiene de procesarlos. La inevitable consecuencia de esta contradicción es una intensa competencia entre los temas" (Zhu, 1992, p. 825). Es decir, los asuntos compiten por ocupar un lugar en la agenda en un juego en el que el ingreso de un 
nuevo issue o el aumento en su frecuencia y jerarquía resulta siempre a expensas de la disminución o, incluso, de la desaparición de otros temas de la superficie mediática.

La operacionalización clásica del concepto de hueco informativo consistió en descontar del espacio total de los ejemplares la publicidad, los avisos clasificados, los anuncios y resultados de carreras de caballos, los listados de cotizaciones bursátiles, las programaciones de radio y televisión, las historietas, los crucigramas y demás entretenimientos. La decisión radicó en que estos contenidos fueron considerados permanentes en los diarios y, por lo tanto, no relevantes para el análisis de las coberturas (Budd, 1964).

El presente trabajo tiene como objetivo analizar críticamente el concepto de hueco informativo. Para ello, además de avanzar en su definición y operacionalización, resulta necesario ponerlo en relación con la noción de relevancia informativa, prestando especial atención a sus diferentes dimensiones: la frecuencia de cobertura y la jerarquía noticiosa. En función de ello, se plantean las siguientes hipótesis y preguntas de investigación, las cuales se sostienen en las definiciones conceptuales vertidas en este apartado y en aportes de trabajos previos (Aruguete, 2011; Budd, 1964; McCombs, 2006; Zunino, 2015).

Hipótesis 1. El conflicto en torno del proceso de implementación de la Resolución No 125/08 obtuvo un importante nivel de relevancia, la cual se evidencia a partir de su frecuencia de cobertura, de la jerarquía ${ }^{2}$ de las noticias vinculadas con el caso y de la porción del hueco informativo que cada diario le asignó al asunto.

Pregunta 1. ¿Cuál fue la relevancia de las piezas periodísticas sobre este caso?

Pregunta 2. ¿Qué porciones del hueco informativo ocuparon las noticias sobre este tema en las agendas de los diarios?

Hipótesis 2. El tamaño del hueco informativo de los periódicos es estable, por lo que el aumento de la relevancia de un asunto resulta siempre a expensas de la presencia y permanencia de otros temas en la agenda mediática.

Pregunta 3. ¿De qué modo se comportó el tamaño de los huecos informativos de los diarios durante el período observado?

Pregunta 4. ¿Qué tipo de relación existe entre los conceptos de hueco informativo y suma cero?

Pregunta 5. ¿Qué factores no ligados al contenido informativo inciden sobre el tamaño y la disposición del news hole?

\footnotetext{
2 El concepto de jerarquía remite a "la visibilidad de la información a partir de su ubicación, su tamaño, su disposición [...] o su mayor frecuencia de cobertura" (Amadeo, 2008, p. 191).
} 


\section{Metodología}

En función de los objetivos específicos y en pos de contrastar las hipótesis, se desarrolló un análisis de contenido cuantitativo sobre los diarios Clarín, La Nación y Página/12 publicados entre el 11 de marzo y el 17 de julio de 2008.

Se define al análisis de contenido como "una técnica de investigación destinada a formular, a partir de ciertos datos, inferencias reproducibles y válidas que puedan aplicarse a su contexto" (Krippendorff, 1990, p. 28).

Se sometieron a observación los 384 ejemplares de los diarios Clarín, La Nación y Página/12 (128 de cada diario) publicados en ese período con el fin de separar las noticias referidas al objeto y de calcular el tamaño del hueco informativo ocupado por estas.

Posteriormente, se recolectaron y se identificaron todos los artículos periodísticos que hicieron referencia explícita al "conflicto del campo" de 2008. Ello permitió obtener una población completa compuesta por 6.266 piezas. Todas fueron relevadas del total de las ediciones impresas que los tres diarios publicaron durante el recorte temporal propuesto (2.322 publicadas por La Nación, 2.157 por Clarín y 1.787 por Página/12).

Dada la extensión del corpus, resultó imprescindible diseñar una muestra aleatoria simple "que sea lo bastante amplia como para contener información suficiente y lo bastante pequeña como para facilitar el análisis" (Krippendorff, 1990, p. 94). El margen de error asociado a estos valores es de 5,1 en más o en menos.

Los diarios cuyas coberturas se analizan constituyen las unidades de contexto de la investigación (Colle, 2011). Clarín y La Nación son los dos periódicos de referencia de la Argentina (Martini, 2007). La Nación fue fundado por el expresidente Bartolomé Mitre en 1870. Es un diario sábana que se caracteriza por una mirada pedagógica de la política y una voluntad orientadora de la clase dirigente que se constituyó históricamente como portavoz de la oligarquía terrateniente (Sidicaro, 1998). Clarín, en tanto, forma parte del mayor multimedios del país. Creado en 1945 por Roberto Noble, es un tabloide multitarget líder en circulación.

La elección de Página/12 se sustenta en otros motivos. Pensado originalmente como un diario de contrainformación (Ulanovsky, 1997), la decisión fundamental que justifica su selección radica en que desde el año 2003 se vio cada vez más alineado en defensa de las políticas del gobierno de Néstor Kirchner, primero, y de las de Cristina Fernández de Kirchner, después (Cremonte, 2010; Koziner \& Zunino, 2013; Zunino, 2013).

\section{Fiabilidad}

Una vez codificadas las notas se realizaron pruebas de fiabilidad. Para ello se construyeron dos submuestras aleatorias del $10 \%$ de las poblaciones totales. Una de ellas 
incluyó 38 ejemplares sobre los cuales se recontabilizó el tamaño del hueco informativo y la otra contempló 36 noticias sobre las que se recodificaron diferentes variables relativas a la estimación de la relevancia del caso. Posteriormente se procedió a cruzar los datos de la codificación original con los de la recodificación. El nivel de acuerdo medio para en el recuento del hueco informativo fue medido mediante el índice de correlación de Spearman ${ }^{3}$ y fue de $\rho .868$, mientras que el acuerdo medio sobre la codificación de la relevancia fue de $\rho$.988. Los datos expresan un alto nivel de fiabilidad.

\section{Resultados}

El tratamiento informativo de la Resolución No 125/08 en los diarios Clarín, La Nación y Página/12 incluyó un total de 6.266 piezas periodísticas que conforman el universo de este trabajo $(N=6.266)$. Estas fueron publicadas en 384 ediciones (128 de cada periódico) entre el 11 de marzo y el 17 de julio de 2008.

El diario La Nación publicó un total de 2.322 piezas sobre el caso, lo que representa un $37,05 \%$ de la población. Clarín, en tanto, incluyó en su agenda 2.157 noticias que significan el 34,43\% del universo. Finalmente, Página/12 le dedicó 1.787 artículos al tema, que alcanza el 28,52\%. Si se analiza el promedio diario de publicación, La Nación dedicó a la resolución mencionada 18,28 notas cada día, Clarín, 16,93 y Página/12, 14,14 .

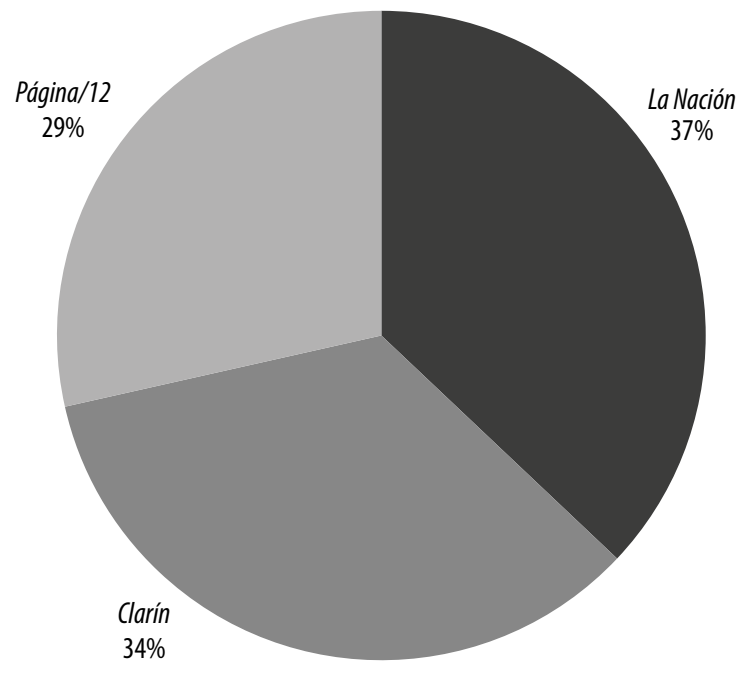

Gráfico 1. Cantidad de piezas periodísticas, según diario (en porcentajes). Cobertura mediática de la Resolución № 125/08. Clarín, La Nación y Página/12. 11 de marzo al 17de julio de 2008.

\footnotetext{
3 El coeficiente de Spearman es una medida de la correlación entre dos variables aleatorias continuas. El indicador oscila entre los valores de -1 y +1 . Un nivel de acuerdo aceptable es cuando el resultado es mayor a Rho de Spearman $=0.7$ (Krippendorff, 1990).
} 
Los resultados expuestos dan cuenta de una alta frecuencia de cobertura y de comportamiento similar entre los tres diarios. Es decir, el volumen de artículos dedicados al "conflicto del campo" fue homogéneo entre Clarín, La Nación y Página/12 durante los 128 días del período.

En función de establecer qué factores influyeron sobre la frecuencia, se la analiza en relación con la variable temporal.

En términos generales, las curvas de frecuencia de los diferentes diarios demuestran un comportamiento similar. Es decir, los picos de cobertura se dieron en las mismas fechas y estuvieron asociados a idénticos hechos ${ }^{4}$. Algunos de ellos -como los enfrentamientos entre simpatizantes del gobierno y de las entidades agropecuarias en las calles y las plazas del país (semana 3), los cortes de ruta y cese de comercialización de granos (semana 7), los actos políticos multitudinarios en provincias del interior (semana 11) y la decisión del gobierno de enviar la norma al Congreso para su discusión

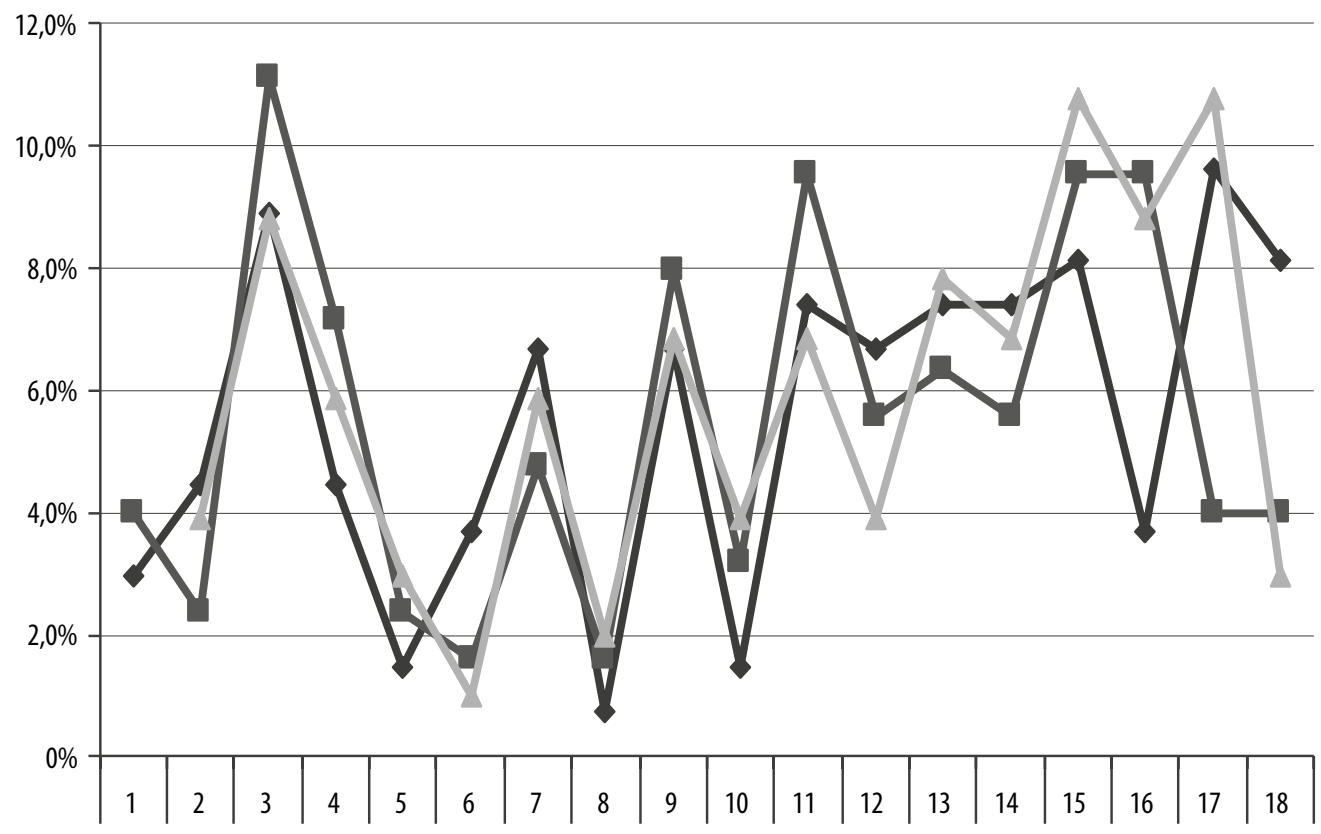

Semana

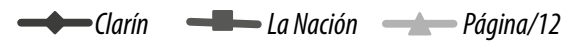

Gráfico 2. Cobertura informativa según semanas del período. Cobertura mediática de la Resolución № 125/08. Clarín, La Nación y Página/12, 11 de marzo al 17de julio de 2008.

\footnotetext{
4 Para un detalle exhaustivo sobre los sucesos referidos en el gráfico ver Barsky \& Dávila (2008) “La rebelión del campo: historia del conflicto agrario argentino".
} 
(semana 15) - elevaron sustancialmente las curvas de frecuencia de cobertura luego del fracaso de las negociaciones.

Los hechos reseñados pueden englobarse dentro de lo que Richard Pride (1995) define como eventos críticos, es decir "acontecimientos dramáticos tales como crisis económicas, desastres ambientales, confrontaciones físicas, iniciativas estratégicas de organizaciones o movimientos sociales o nuevas políticas públicas" (Pride, 1995, p. 5). Este tipo de acontecimientos constituye discontinuidades radicales en el acontecer público que contribuyen con la definición social de los problemas.

La constatación de que el nivel de atención mediática se intensificó producto de este tipo de sucesos permite vislumbrar que el desarrollo de eventos críticos repercute de manera directa en la frecuencia de cobertura de los diarios. Este hallazgo se torna más elocuente si se tiene en cuenta que según los datos proporcionados en el gráfico 2 las curvas de frecuencia de los diferentes diarios resultaron congruentes tanto en sus sentidos como en las fechas en las que se dieron sus picos máximos.

Ahora bien, el análisis de la frecuencia de manera descontextualizada no permite concluir a priori que el diario que mayor cantidad de notas le dedica a un issue

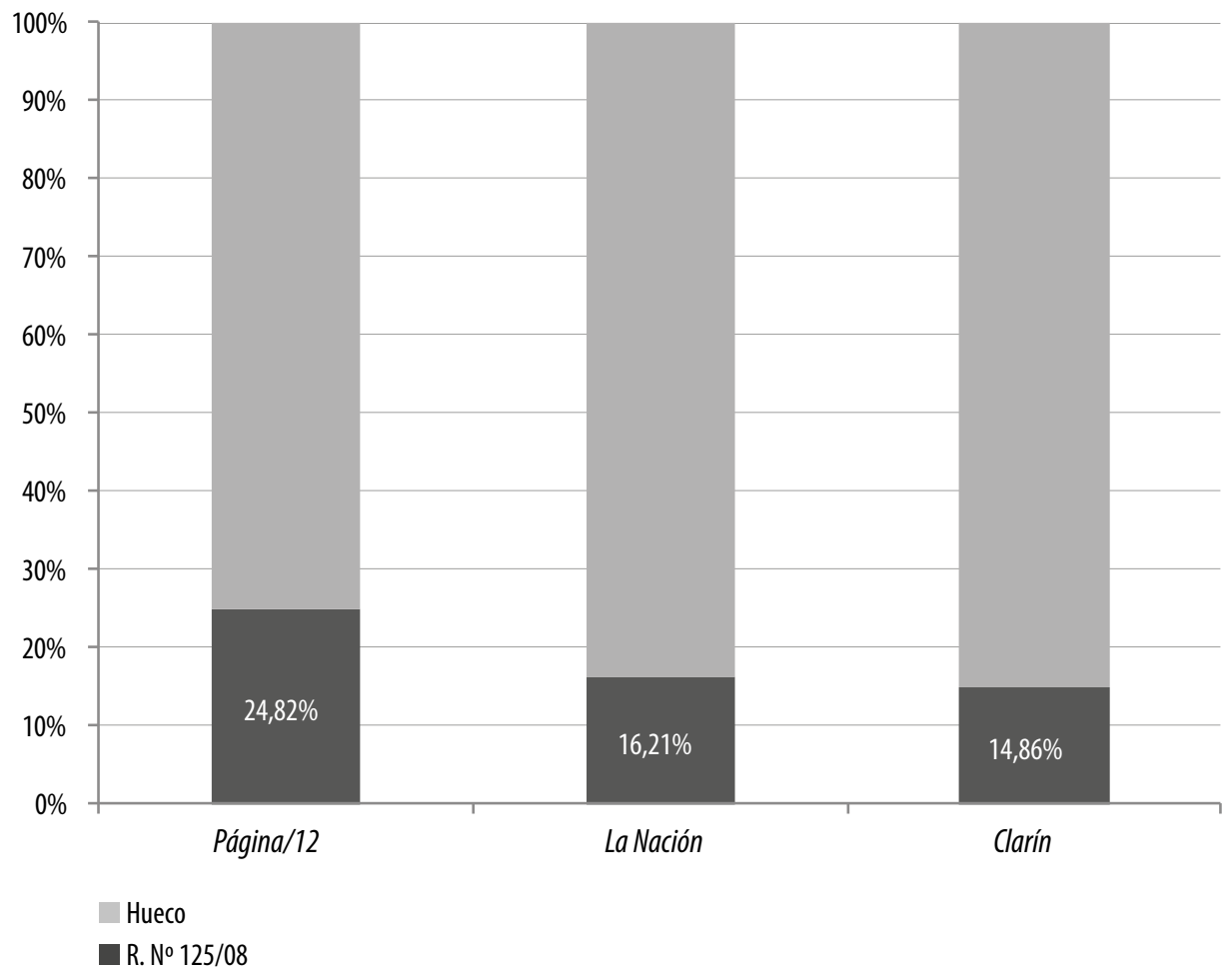

Gráfico 3. Relación entre hueco informativo y frecuencia de cobertura según diario. Cobertura mediática de la Resolución No 125/08. Clarín, La Nación y Página/12, 11 de marzo al 17de julio de 2008. 
es el que más importancia total le da. En función de los objetivos específicos de este trabajo resulta aconsejable medir la frecuencia en relación con el concepto de hueco informativo.

Para calcular la porción del hueco informativo que ocupó el "conflicto del campo" en cada periódico se contabilizó la totalidad de las noticias publicadas, independientemente de los temas a los que refirieran, y se separaron las que hicieron mención explícita al caso.

Durante los 128 días que duró el conflicto, el diario La Nación editó un total de 14.324 piezas periodísticas, de las cuales 2.322 refirieron al caso, lo que representó el $16,21 \%$ de su hueco informativo.

En el mismo lapso, Clarín publicó un total de 14.513 piezas, de los cuales 2.157 tuvieron como tema central el "conflicto campo-gobierno". Es decir, la cobertura del tema que aquí se analiza ocupó un 14,86\% del hueco informativo del diario.

En tanto, Página/12 incluyó en su agenda 7.240 piezas periodísticas durante el período, de las cuales 1.787 tuvieron como tema central a la Resolución No 125/08, cifra que representa un $24,82 \%$ del news hole.

Los datos demuestran que si bien la frecuencia de cobertura fue mayor en $\mathrm{La}$ Nación y en Clarín en términos absolutos, Página/12 le dio más importancia al tema, puesto que este ocupó un porcentaje mayor de su hueco informativo.

Adicionalmente, resulta importante analizar la evolución temporal de la cobertura noticiosa según el hueco informativo de cada diario. El gráfico 4 expresa la relación entre ambas variables.

A simple vista se puede apreciar que el news hole de Clarín y La Nación es estable, cercano a la línea de las 800 piezas semanales. En cambio, el de Página/12 tiene aproximadamente la mitad de ese tamaño. En tanto, las curvas de frecuencia de cobertura de los tres diarios no solo alcanzaron picos y depresiones en los mismos momentos, sino que además resultaron muy cercanas entre sí. Esto significa que, a pesar de que Página/12 edita menor cantidad de noticias diarias, la frecuencia de cobertura del "conflicto del campo" se mantuvo para este diario en niveles parecidos a los de Clarín y La Nación, lo que revela la mayor trascendencia que este medio le otorgó al tema.

Un interrogante central para los intereses de este estudio es si los picos de cobertura de la Resolución № 125/08 implicaron un aumento del hueco informativo. Es decir, si la edición de una mayor cantidad de noticias sobre el tema significó que el espacio disponible para la información creciera. La respuesta a este interrogante es negativa para los tres diarios analizados. En efecto, una mayor cantidad de notas dedicadas al caso no implicó nunca que los periódicos decidieran incorporar páginas a sus ejemplares para ampliar el espacio disponible para las noticias. 
Esta constatación empírica permite responder una de las preguntas de investigación de este estudio acerca de la relación existente entre los conceptos de hueco informativo y suma cero. La falta de elasticidad de los huecos informativos, aun en momentos de alta frecuencia de un tema, permite inferir que una mayor dedicación de espacio al conflicto del campo siempre se dio a expensas de la salida o la disminución de la importancia de otros temas en la superficie mediática. Es decir, se verifica que al no haberse expandido el news hole durante el tratamiento de un caso con altos niveles de cobertura es probable que otros issues hayan quedado fuera de agenda o relegados a sitios marginales.

Ahora bien, ¿en qué contextos los diarios incorporan páginas a sus cuerpos principales? ¿Y qué influencias tiene esta decisión sobre el hueco informativo?

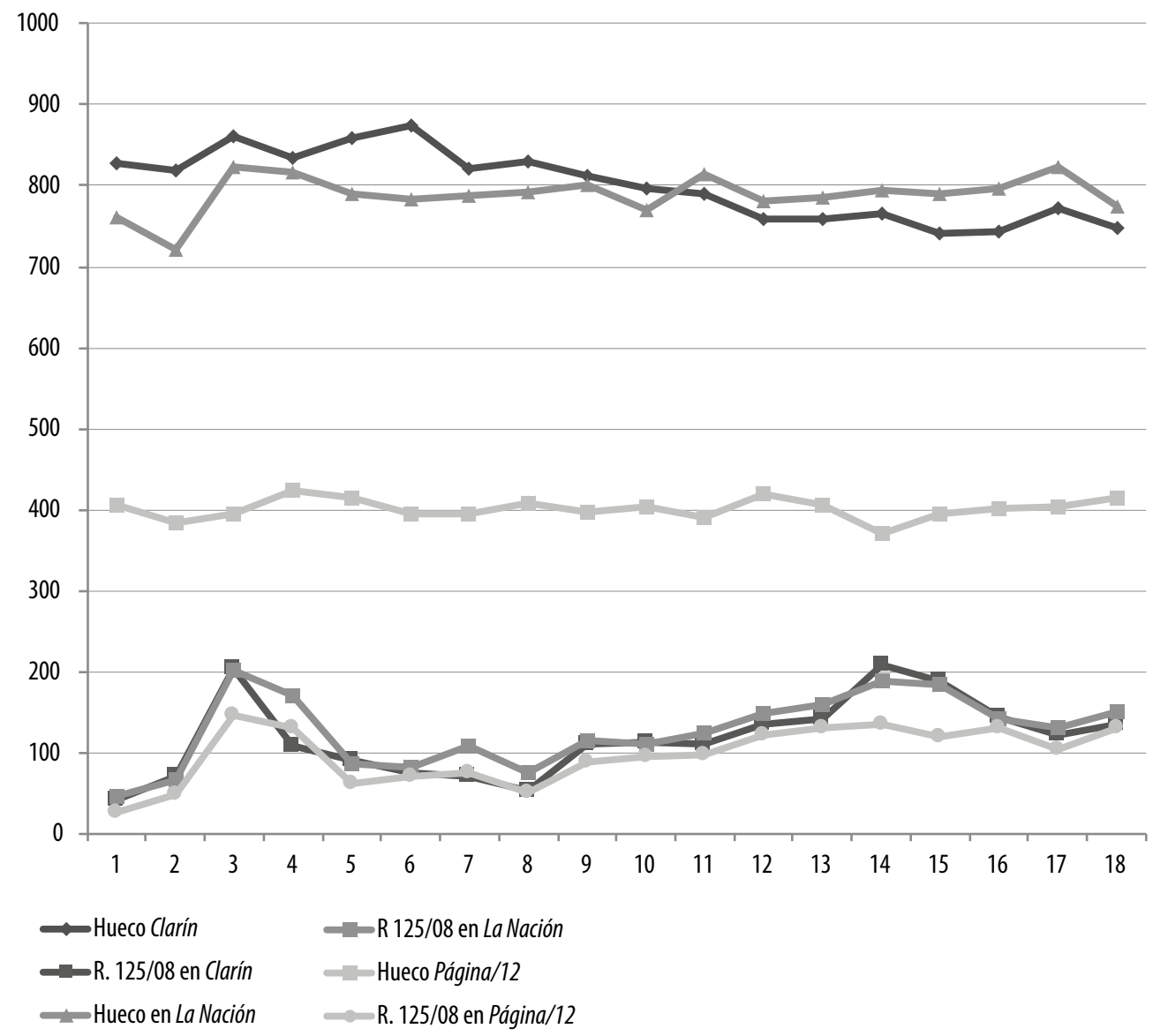

Gráfico 4. Evolución temporal de la frecuencia de cobertura de la Resolución № 125/08 según hueco informativo. Clarín, La Nación y Página/12, 11 de marzo al 17de julio de 2008. 
Uno de los elementos dejados fuera de consideración analítica en la operacionalización clásica del hueco informativo fueron las publicidades. Por ser consideradas elementos estables de los ejemplares, tales como las secciones fijas de chistes, entretenimientos y carreras, no se han hallado investigaciones previas que analizaran de manera interdependiente la disposición de contenidos publicitarios e informativos.

Con el fin de explorar qué tipo de factores operan sobre la cantidad de páginas que los diarios deciden incorporar a sus ejemplares, en primer término se analiza el impacto de la variable temporal sobre el tamaño promedio de los diarios.

El diario Clarín es el que más modificó la cantidad de páginas de su hueco informativo durante el período observado. Mientras que los lunes tiene un promedio de 46 páginas, permanece estable entre 42 y 48 de martes a jueves, asciende a 66 los viernes y sube a 68 los sábados para retroceder a 64 los domingos. Un comportamiento similar evidenció el diario La Nación. Comienza la semana con un promedio de 26 páginas que ascienden a 32 los jueves y los viernes, para subir a 42 los sábados y a 48 los do-

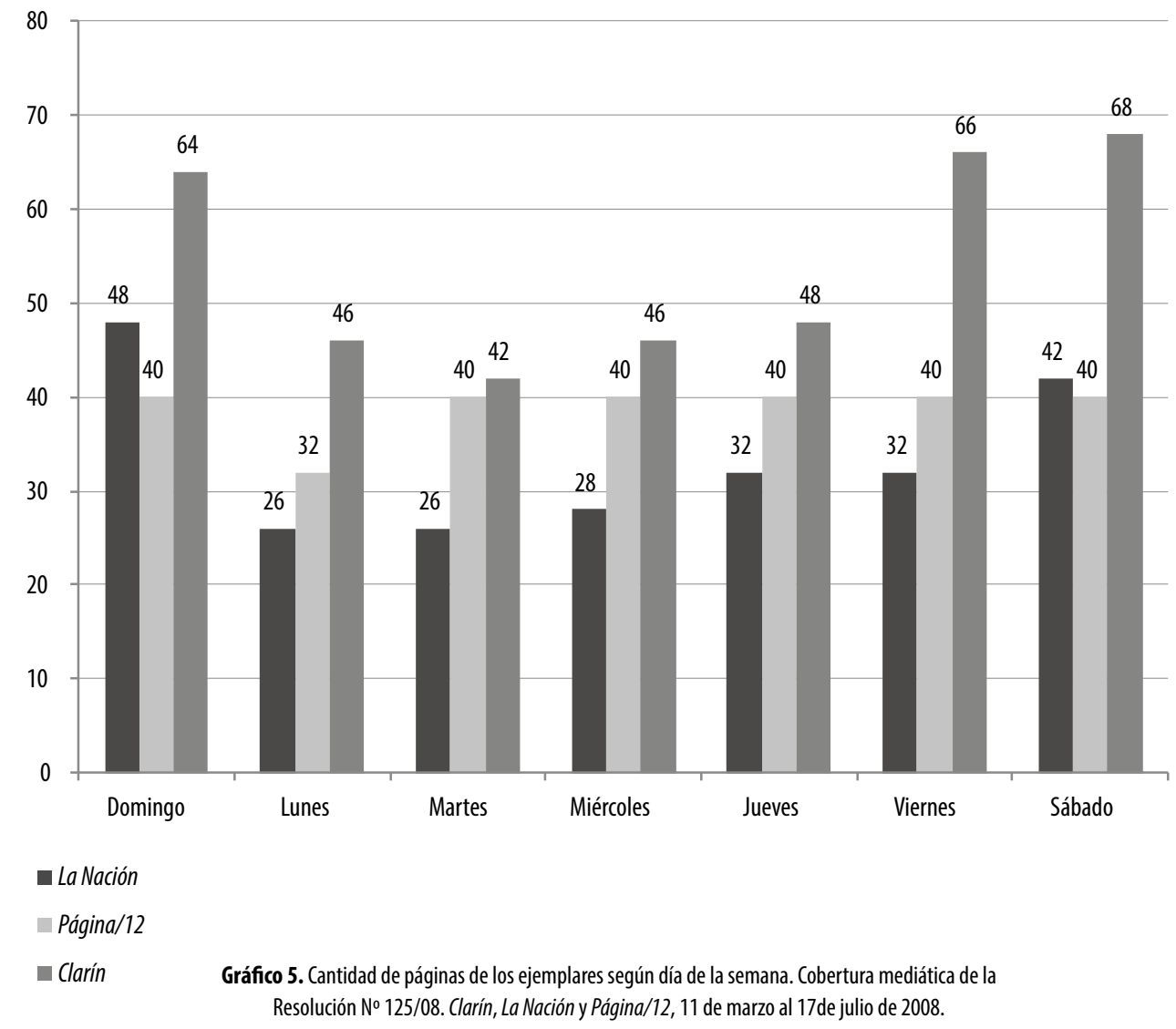


mingos ${ }^{5}$. En tanto, Página/12 mostró un patrón diferente, ya que el tamaño de sus ejemplares permanece estable en torno a las 40 páginas de martes a domingo, cayendo a 32 los lunes.

La coincidencia en Clarín y La Nación de un aumento de la cantidad de páginas en los días de mayor circulación ${ }^{6}$ resulta evidente. Y este comportamiento puede ser explicado no por el aumento del hueco informativo, dado que como se vio en el gráfico 2 este permaneció estable durante 128 días, sino por una importante anexión de más y mayores publicidades en este tipo de días. El comportamiento diferente de Página/127, que evidenció ejemplares de tamaño más homogéneo durante toda la semana, se debe a que este diario se financia principalmente con publicidad oficial, la cual además de ser más escasa que la privada, es más estable.

Con el fin de aportar sustento empírico a las afirmaciones precedentes se decide medir del mismo modo como se hizo con el hueco informativo al espacio que se denominará hueco publicitario, es decir, la superficie de los ejemplares dedicada al emplazamiento de publicidades, ya sean públicas, privadas, comerciales y/o de bien público.

Durante el período estudiado Clarín incluyó en sus ediciones ${ }^{8}$ un total de 6.383 avisos publicitarios. Entre ellos, 2.207 fueron pequeños ${ }^{9}$ y se ubicaron en página par; 296 fueron grandes ${ }^{10} \mathrm{y}$ se ubicaron en página par; 2.247 fueron pequeños y se ubicaron en página impar, y 1.461 fueron grandes y se ubicaron en página impar.

En el mismo lapso, La Nación publicó un total de 7.340 avisos: 3.066 fueron pequeños y se ubicaron en página par; 484 grandes en página par; 2.984 pequeños en página impar y 722 grandes en página impar.

En tanto, Página/12 incluyó un total de 1.561 publicidades en el período observado. De ellas, 591 fueron pequeñas y se ubicaron en página par; 122 fueron grandes y se ubicaron en página par; 585 fueron pequeñas y se ubicaron en página impar, y 249 fueron grandes y se ubicaron en página impar.

Ahora bien, en pos de enriquecer el análisis sobre el comportamiento del hueco publicitario se midió su evolución temporal según tamaño y tipo de página dispuesto para este tipo de contenidos. La tabla 1 expresa los resultados.

\footnotetext{
La menor cantidad de páginas en La Nación se compensa por su tamaño, ya que es el único de los tres diarios analizados que mantiene un formato "sábana".

6 Clarín tiene un promedio de circulación de 155.606 ejemplares de lunes a viernes, que sube a 244.002 los sábados y a 492. 670 los domingos. El promedio de La Nación es de 89.148 ejemplares de lunes a viernes, que asciende a 183.455 los sábados y a 304.009 los domingos. Fuente: Instituto Verificador de Circulaciones, febrero de 2016. Disponible en: http://www.revistaairelibre.com.ar/boletin.ivc.pdf.

7 El diario Página/12 no es auditado por el IVC, pero según datos de la Sociedad de Distribuidores de Diarios, Revistas y Afines este promedia los 10.000 ejemplares de lunes a viernes, y asciende a 15.000 los sábados y a 20.000 los domingos. 8 Se contaron las publicidades incluidas en las mismas secciones que componen el hueco informativo. Es decir, no se tuvieron en cuenta los suplementos especiales, chistes $\mathrm{u}$ otros entretenimientos.

9 Se consideró avisos pequeños a los menores a media página.

10 Se consideró avisos de gran tamaño a los iguales o mayores a media página.
} 
Los datos de la tabla 1 evidencian que tanto en Clarín como en La Nación los sábados y domingos las publicidades de gran tamaño ubicadas en página impar, las más visibles de los diarios, tienden a duplicar el promedio semanal. Si se tiene en cuenta que en el caso de Clarín llegan a publicarse entre 14 y 20 anuncios de más de media página esos días y en La Nación entre 9 y 11, resulta evidente que el drástico aumento de páginas de los ejemplares en este tipo de días se debe a la anexión de publicidad y no de espacio para la información. El comportamiento diferencial de Página/12 no hace más que confirmar la afirmación anterior. En un diario en el que el recurso publicitario permanece estable toda la semana no resulta necesario incorporar páginas a los ejemplares.

El estudio del comportamiento del tamaño de los ejemplares permite poner en discusión una cuestión central. En tres medios de comunicación cuya función central es informar, y en el contexto de un caso que insumió importantes niveles de los huecos informativos, el espacio dedicado a las noticias nunca aumentó, mientras que el que se dedicó a la publicidad, principal mecanismo de financiamiento de las empresas periodísticas, sí lo hizo en los días de mayor circulación.

La afirmación precedente es coherente con las características estructurales de la prensa como rama específica de las industrias culturales. El modelo de negocios de la prensa gráfica se denomina modelo de doble mercado y consiste en la financiación mediante la venta de ejemplares - por suscripción y en los puntos de venta- y por intermedio de anunciantes publicitarios (Albornoz, 2006). Según Zallo (1998), se trata de una industria que comprende a una mercancía compleja, puesto que incluye infor-

Tabla 1. Cantidad de avisos publicitarios según tamaño, diario y día de la semana. Cobertura mediática de la Resolución № 125/08. Clarín, La Nación y Página/12, 11 de marzo al 17de julio de 2008.

\begin{tabular}{|c|c|c|c|c|c|c|c|c|}
\hline & & Lunes & Martes & Miércoles & Jueves & Viernes & Sábado & Domingo \\
\hline & Par chica & 21,11 & 15,55 & 15,83 & 16,44 & 13,94 & 13,22 & 25,72 \\
\hline & Par grande & 1,44 & 1,94 & 1,79 & 1,66 & 1,38 & 4 & 4,11 \\
\hline & Impar chica & 20,38 & 18,55 & 18,26 & 21,77 & 13,55 & 8,72 & 22,55 \\
\hline \multirow[t]{4}{*}{ Clarín } & Impar grande & 10,27 & 7,77 & 8,95 & 9,05 & 10,5 & 20 & 14,11 \\
\hline & Par chica & 16,39 & 23,27 & 21,05 & 19 & 21,5 & 21,83 & 46,11 \\
\hline & Par grande & 2,16 & 2,44 & 2,42 & 3,33 & 3,33 & 6,55 & 6,5 \\
\hline & Impar chica & 11,05 & 17,16 & 17,47 & 24,33 & 15 & 19,61 & 60,16 \\
\hline \multirow[t]{4}{*}{ La Nación } & Impar grande & 2 & 3,5 & 3,42 & 4,88 & 5,66 & 11,38 & 9,05 \\
\hline & Par chica & 5,18 & 4,66 & 4,83 & 4,5 & 5,41 & 4,22 & 5,17 \\
\hline & Par grande & 0,25 & 0,61 & 0,55 & 0,39 & 0,29 & 0,38 & 0,52 \\
\hline & Impar chica & 3,81 & 5,11 & 4,5 & 4,44 & 4,65 & 6,55 & 5,82 \\
\hline Página/12 & Impar grande & 1 & 1,66 & 1,83 & 2,33 & 2,88 & 2,5 & 2 \\
\hline
\end{tabular}


maciones diversas y múltiples de las que el lector solo consume el 10\%. Es decir, las páginas más leídas y en las que se incluye la publicidad más cara sostienen el resto de la información.

En función de los hallazgos descriptos es posible establecer, entonces, una nueva hipótesis: el hueco informativo y el hueco publicitario no constituyen compartimentos estancos, sino que se influyen y se condicionan entre sí. Es decir, resulta plausible que una mayor cantidad de avisos publicitarios en los sitios más relevantes del diario vaya en detrimento de la ubicación de noticias relevantes en los sitios de mayor visibilidad. Para analizar esta problemática resulta necesario estudiar qué tipo de relación existe entre estos dos espacios interdependientes.

En un trabajo previo se había constatado que las áreas de mayor visibilidad tienden a coincidir con aquellas en los que se da una mayor competencia temática (Zunino, 2015). Estos son las primeras planas y las páginas impares de los diarios.

Para analizar la jerarquía de la Resolución No 125/08 en Clarín, La Nación y Página/12 se midieron diez variables complementarias habitualmente utilizadas en trabajos de este tipo (McCombs \& Shaw, 1972; Aruguete, 2011; Budd, 1964; Gutiérrez Coba, 2001; Kiousis \& McCombs, 2004; Igartua, Muñiz \& Cheng, 2005).

La tabla 2 permite realizar dos afirmaciones importantes. La primera de ellas es que el caso bajo estudio alcanzó un nivel alto de jerarquía informativa. Una gran cantidad de piezas de gran tamaño, acompañadas de imágenes, con titulares llamativos, ubicadas en la mitad superior de las páginas y con la firma de sus autores, así lo expresan. Asimismo, las piezas con valencia definida, tal como lo afirma Kiousis (2004), también realzan la importancia del caso, puesto que las notas con mayor cantidad de elementos afectivos, especialmente negativos, al aumentar el dramatismo colocan al issue al tope de la agenda.

Ahora bien, si se observa qué sucedió en los sitios de mayor competencia temática, como las portadas y las páginas impares, es posible advertir que los porcentajes de presencia de notas sobre el tema bajaron considerablemente. Una primera explicación para que esto haya sucedido remite a lo acotado de estos espacios.

En un caso como el que se analiza, en el cual la frecuencia de publicación de noticias fue muy alta, es probable que no todas ellas puedan ubicarse en los sitios más visibles, y llegaron a competir incluso entre sí mismas.

Sin embargo, otro dato que no se puede soslayar es la presión que ejercen los avisos en este tipo de espacios. Las tapas de Clarín y La Nación se componen de un promedio de 11 titulares diarios, mientras que la de Página/12 se estructura sobre la base de un solo título destacado al que lo rodea una media de siete recuadros más pequeños. Las notas referidas al "conflicto del campo" que penetraron en las primeras planas alcanzaron el 21,5\% en Clarín, el 17,5\% en La Nación y el 32,4\% en Página/12. Si bien estos 
porcentajes evidencian una alta presencia del tema en este lugar central, es necesario contextualizar estos datos en el marco de una competencia que excede a los asuntos noticiables.

Se pudo constatar que Clarín publicó un promedio de 1,25 publicidades diarias en tapa, Página/12, 0,65 y La Nación, 0,73. Este tipo de anuncios, generalmente dedicados a publicitar productos incluidos con las ediciones de los mismos diarios, aunque también bienes y servicios externos, compitieron con las noticias en el lugar de mayor visibilidad de la agenda.

Algo similar sucedió con la presencia del caso en las páginas impares, las más visibles de los periódicos (McCombs, 2006). La explicación de por qué la mayoría de las

Tabla 2. Jerarquía. Cobertura mediática de la Resolución № 125/08. Clarín, La Nación y Página/12, 11 de marzo al 17de julio de 2008.

\begin{tabular}{lccc}
\hline & \multicolumn{3}{c}{ Diario } \\
\hline Presencia en tapa $^{11}$ & Clarín & La Nación & Página/12 \\
\hline Apertura de sección $^{12}$ & $21,50 \%$ & $17,50 \%$ & $32,40 \%$ \\
\hline Página impar $^{13}$ & $14,10 \%$ & $14,30 \%$ & $12,70 \%$ \\
\hline Valencia definida $^{14}$ & $43,70 \%$ & $47,60 \%$ & $48,00 \%$ \\
\hline Presencia de imagen $^{15}$ & $70,40 \%$ & $73,00 \%$ & $79,40 \%$ \\
\hline Gran tamaño $^{16}$ & $63 \%$ & $52,90 \%$ & $63,90 \%$ \\
\hline Titulares grandes $^{17}$ & $63 \%$ & $61,10 \%$ & $56,90 \%$ \\
\hline Mitad superior $^{18}$ & $60,00 \%$ & $42,10 \%$ & $51 \%$ \\
\hline Notas firmadas $^{19}$ & $73,30 \%$ & $66,70 \%$ & $63,70 \%$ \\
\hline Presencia de bajada $^{20}$ & $63,70 \%$ & $69,80 \%$ & $54,90 \%$ \\
\hline
\end{tabular}

1 La importancia de esta variable radica en que "las noticias de portada del diario tienen unas dos veces más lectores que las que aparecen en las páginas interiores” (McCombs, 2006, p. 107).

2 Las noticias que abren sección son consideradas como las más destacadas (McCombs \& Shaw, 1972).

3 Los artículos dispuestos en página impar adquieren mayor visibilidad y, por lo tanto, resultan más importantes en la cobertura informativa (Aruguete, 2011; Budd, 1964; Igartua et al., 2005).

4 Los artículos periodísticos con elementos afectivos explícitos, sobre todo negativos, adquieren mayor visibilidad que los restantes (Kiousis, 2004).

5 Se parte de la premisa de que las noticias “que llevan gráficos atractivos atrapan la atención de más lectores” (McCombs, 2006, p. 107).

6 Se consideran de gran tamaño a las piezas mayores a media página en Clarín y Página/12 y a un cuarto de página en La Nación. El tamaño de las piezas les aporta visibilidad dentro de la agenda mediática (Budd, 1964).

7 El tamaño de los titulares también es un indicador de la jerarquía informativa (McCombs \& Shaw, 1972). Se consideraron titulares grandes a los mayores a la mitad del ancho de página en los tres diarios relevados.

8 Los artículos que se incluyen en la parte superior de la página son más visibles que los que aparecen en la inferior (Budd, 1964; Gutiérrez Coba, 2001).

9 La firma de la nota denota la importancia que le otorga el medio al caso (Aruguete, 2011; Casermeiro de Pereson, 2004; Odriozola Chené, 2012).

10 Se sostiene que "las notas periodísticas que llevan entradilla se consideran como las más destacadas" (Gutiérrez Coba, 2001, p. 10). 
noticias referidas al caso se ubicaron en las páginas pares (56,3\% en Clarín, 52,4\% en La Nación y $52 \%$ en Página/12) incluye varias aristas. La primera de ellas es que las piezas de mayor tamaño se ubicaron en página impar, lo que quita espacio al resto de las informaciones. Sin embargo, no debe dejarse de mencionar que las páginas impares, sobre todo los días de mayor circulación, estuvieron ocupadas en buena medida por avisos publicitarios.

Entonces, la disposición de las noticias en este tipo de sitios se vio condicionada por factores endógenos y exógenos al ámbito informativo. Respecto de los primeros, la competencia temática se hizo encarnizada en los sitios más visibles. Respecto de los segundos, resulta imperioso afirmar que el contenido publicitario no resulta inocuo al contenido informativo. La tendencia a incluir mayor cantidad de noticias en páginas pares se debe a una también mayor cantidad de avisos de gran tamaño en las páginas impares, sobre todo los fines de semana.

En función de los hallazgos descriptos, se propone entonces considerar el hueco informativo y el hueco publicitario como dos espacios interrelacionados que conforman un espacio o hueco total en el que se incluyen informaciones y publicidades: dos contenidos centrales para el sostenimiento económico de este tipo de medios. La importancia relativa de ambos contenidos respecto del caso que se analiza se expresa en el gráfico 6.

Si se tienen en cuenta la cantidad de notas periodísticas y la totalidad de piezas publicitarias se puede establecer la importancia relativa de los huecos informativos y

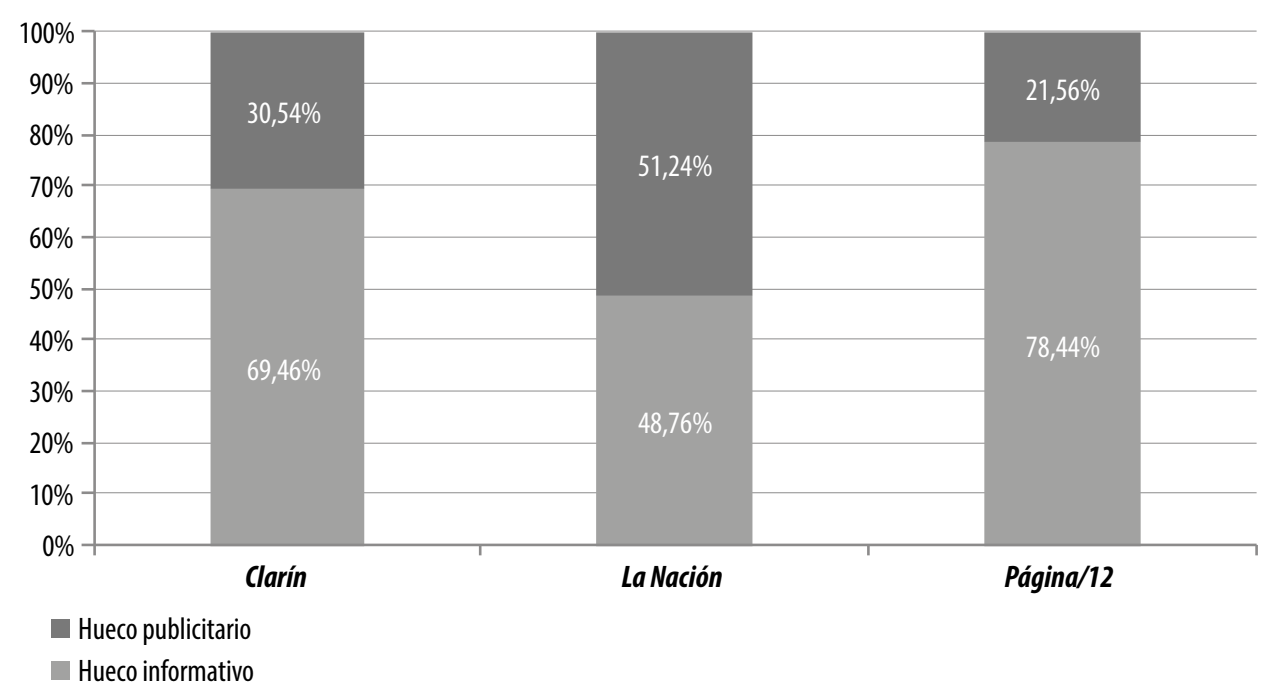

Gráfico 6. Hueco informativo y hueco publicitario. Cobertura mediática de la Resolución № 125/08. Clarín, La Nación y Página/12, 11 de marzo al 17de julio de 2008. 
publicitarios de cada diario. El periódico que mayor cantidad de publicidad incluyó en sus ejemplares fue La Nación, lo que corresponde a más de la mitad del contenido $(51,24 \%)$ de este tipo. Este diario posee una diferencia respecto de los demás porque incluye avisos clasificados de diversos rubros en su cuerpo central, intercalados con las piezas periodísticas. En tanto, más de un tercio de la superficie de Clarín correspondió a publicidades (30,54\%). El porcentaje fue menor en Página/12 (21,56\%).

Finalmente, resulta revelador que mientras el hueco informativo permaneció estable durante todos los días de la semana, el hueco publicitario tendió a ser mucho más flexible, y aumentó drásticamente su tamaño los sábados y domingos. Este dato, que resulta ilustrativo de los modos de financiamiento de las empresas periodísticas, no debe soslayar un doble estándar del comportamiento de estas respecto de sus dos contenidos centrales: mientras que en el contexto de un caso fuertemente resonante para la ciudadanía argentina los diarios nunca decidieron incorporar páginas a sus ejemplares para satisfacer la demanda informativa de un asunto que tendió a opacar y a excluir a otros temas de la agenda mediática, la incorporación de pliegos a los ejemplares resultó habitual para satisfacer la demanda de los anunciantes, actores centrales del financiamiento de la actividad periodística.

\section{Conclusiones}

En el presente estudio se pretendió sistematizar y operacionalizar el concepto de hueco informativo (Budd, 1964). Concebido como la cantidad de espacio total dispuesto para la información una vez descontadas las secciones fijas de los ejemplares como las carreras, chistes, obituarios y publicidad, este concepto habitualmente fue utilizado de manera descontextualizada de otros como la relevancia informativa y la disposición de los avisos publicitarios en el espacio total de los ejemplares.

El desafío de este estudio consistió en poner en relación dimensiones de análisis que habitualmente se presentan como inconexas. En ese sentido, los resultados del trabajo empírico presentaron hallazgos relevantes en dos dimensiones. La primera de ellas se relaciona con el caso de estudio sobre el que se pretendía conocer su relevancia en función de su frecuencia de cobertura y su jerarquía. Respecto de este nivel de análisis, se evidenció una gran cantidad de notas publicadas por los tres diarios, dispuestas jerárquicamente en sitios altamente visibles y que ocuparon porciones considerables de los huecos informativos.

Un segundo nivel de análisis abarcó interrogantes teóricos acerca del comportamiento del hueco informativo y sus condicionantes. En el trabajo se estimó que los contenidos publicitarios no podían ser considerados inocuos en empresas periodísticas que se valen de ellos para su sostenimiento. 
Los hallazgos en este nivel de indagación revelaron que, mientas que el hueco informativo en tres diarios con líneas editoriales, alcances y modos de financiamiento diversos resultaron poco flexibles, aun en un contexto en el que la demanda informativa fue muy alta, la cantidad de avisos tendió a incrementarse en los días de mayor circulación, y generaron la anexión de espacio físico a los ejemplares. Es decir, mientras que ningún acontecimiento informativo, base de la función de vigilancia del entorno de los medios de comunicación (Lazarsfeld \& Merton 1948; Wolf, 1987; McQuail, 1992; Wright, 1959), justificó la incorporación de páginas a los ejemplares, la inclusión de una mayor cantidad de publicidad tornó habitualmente flexible al espacio dedicado a ella.

Dado este comportamiento evidenciado en el trabajo empírico, se decidió otorgar la denominación de hueco publicitario al espacio destinado por los medios de comunicación a la disposición de anuncios publicitarios comerciales y/o de bien público con financiamiento público y/o privado.

La constatación de que habitualmente este tipo de contenido es ubicado en páginas impares - las más visibles de los diarios-, e incluso en las portadas, hace suponer que el hueco informativo se ve condicionado por la disposición de avisos publicitarios. Las evidencias sostienen la afirmación de que los bajos porcentajes de piezas sobre la Resolución No 125/08 ubicadas en primera plana y páginas impares se explica no solo por la competencia temática hacia adentro del hueco informativo sino también por la competencia con el contenido publicitario en estos espacios de alta visibilidad.

En un contexto de alta conflictividad social, a raíz de un caso de gran impacto económico y político, los medios analizados nunca decidieron compensar el espacio dedicado a la Resolución No 125/08 con una mayor cantidad de páginas tendientes a incluir al resto de los acontecimientos trascendentes para la vida social en sus agendas. En cambio, la demanda publicitaria fue satisfecha asiduamente, incluso otorgando a los anunciantes el espacio más visible de cada ejemplar. Este aspecto, que deberá ser investigado en diferentes contextos e incluso en diversos tipos de soportes, deja entrever un dato que no debiera pasar inadvertido: la primacía del criterio económico por sobre el periodístico en las empresas.

Esta constatación, que no hace más que corroborar el condicionamiento que le impone el material publicitario al informativo, debería incentivar la formulación de interrogantes acerca de la relación entre financiamiento y contenido periodístico. Relación antigua y espinosa sobre la que es menester indagar empíricamente.

\section{Referencias}

Albornoz, L. (2006). Periodismo digital: los grandes diarios en la red. Buenos Aires: Ediciones La Crujía. 
Amadeo, B. (2008). Framing: modelo para armar. En: Baquerín de Riccitelli, M. T. (ed.). Los medios ¿aliados o enemigos del público? (p. 183-281). Buenos Aires: EDUCA.

Amado, A. \& Rotelli, N. (2011). La investigación de medios en situaciones de conflicto: supuestos y aportes. Ponencia presentada en el $10^{\circ}$ Congreso nacional de ciencia política, "Democracia, integración y crisis en el nuevo orden global: tensiones y desafíos para el análisis político". Córdoba: Sociedad Argentina de Análisis Político.

Aruguete, N. (2011). Los medios y la privatización de ENTel. Berlin: Editorial Académica Española.

Aruguete, N. (2015). El poder de la agenda: política, medios y público. Buenos Aires: Biblos.

Barsky, O. \& Dávila, M. (2008). La rebelión del campo: historia del conflicto agrario argentino. Buenos Aires: Sudamericana.

Barsky, O. \& Gelman, J. (2009). Historia del agro argentino: desde la conquista hasta comienzos del siglo XXI. Buenos Aires: Sudamericana.

Becerra, M. \& López, S. (2009). La contienda mediática: temas, fuentes y actores en la prensa por el conflicto entre el gobierno y las entidades del campo argentino en 2008. Revista de ciencias sociales, 2da. época, 16, p. 9-30.

Brosius, H. B. \& Kepplinger, H. M. (1992). Beyond agenda-setting: the influence of partisanship and television reporting on the electorate's voting intentions. Journalism \& Mass Communication Quarterly, 69 (4), p. 893-901.

Budd, R. W. (1964). U.S. news in the press down under. Public opinion quarterly, 28 (1), p. 39-56.

Casermeiro de Pereson, A. (2004). Los medios en las elecciones: la agenda setting en la Ciudad de Buenos Aires. Buenos Aires: EDUCA.

Colle, R. (2011). El análisis de contenido de las comunicaciones (9ºd.). La Laguna: Sociedad Latina de Comunicación Social.

Cremonte, J. P. (2010). Cada cual atiende su juego: la construcción del conflicto entre el Gobierno Nacional y las entidades agropecuarias en Clarín, La Nación y Página/12. En: Aronskind, R. \& Vommaro, G. (eds.). Campos de batalla: las rutas, los medios y las plazas en el nuevo conflicto agrario (p. 227-266). Buenos Aires: Prometeo; Los Polvorines: Universidad Nacional de General Sarmiento.

Dader, J. L. (1992). La canalización o fijación de la "agenda" por los medios. En: A. Muñoz Alonso, A.; Monzón, J. I.; Rospir, J. \& Dader, J. L. (eds.). Opinión pública y comunicación política (p. 294-318). Madrid: EUDEMA.

Gutiérrez Coba, L. (2001). Temas de interés para los periódicos de prestigio: el caso de El País (España ) y El Espectador (Colombia ). ZER, 6 (1), p. 131-157. 
Igartua, J. J., Muñiz, C. \& Cheng, L. (2005). La inmigración en la prensa española: aportaciones empíricas y metodológicas desde la teoría del encuadre noticioso. Migraciones, 17, p. 143-181.

Kiousis, S. (2004). Explicating media salience: s factor analysis of New York Times issue coverage during the 2000 U. S. presidential election. Journal of communication, 54 (1), p. 71-87.

Kiousis, S. \& McCombs, M. (2004). Agenda-setting effects and attitude strength: political figures during the 1996 presidential election. Communication research, 31 (1), p. 36-57.

Koziner, N. \& Zunino, E. (2013). La cobertura mediática de la estatización de YPF en la prensa argentina: un análisis comparativo entre los principales diarios del país. Global media journal México, 10 (19), p. 1-25.

Krippendorff, K. (1990). Metodología de análisis de contenido: teoría y práctica. Buenos Aires: Paidós.

Lazarsfeld, P. \& Merton, R. (1948). Comunicación de masas, gusto popular y acción social organizada. En: Muraro, H (ed.). La comunicación de masas (p. 77-93). Buenos Aires: Centro Editor de América Latina.

Martini, S. (2007). Argentina, prensa gráfica, delito e inseguridad. En: Rey, G. (ed.). Los relatos periodísticos del crimen (p. 21-54). Bogotá: Friedrich-Ebert-Stiftung; Centro de Competencia en Comunicación para América Latina.

McCombs, M. (1992). Explorers and surveyors: expanding strategies for agendasetting research. Journalism \& mass communication quarterly, 69 (4), p. 813-824. McCombs, M. (2006). Estableciendo la agenda: el impacto de los medios en la opinión pública y el conocimiento. Barcelona: Paidós Ibérica.

McCombs, M. \& Shaw, D. (1972). The agenda-setting function of the mass media. Public opinion quarterly, 36, p. 176-187.

McQuail, D. (1992). La acción de los medios: los medios de comuniación y el interés público. Buenos Aires: Amorrortu.

Novaro, M.; Bonvechi, A. \& Cherny, N. (2014). Los límites de la voluntad: los gobiernos de Duhalde, Néstor y Cristina Kirchner. Buenos Aires: Ariel.

Odriozola Chené, J. (2012). Cibermedios y “agenda-setting”: la configuración de la agenda mediática internacional. Estudios sobre el mensaje periodístico, 18 (1), p. 157-171.

Pride, R. A. (1995). How activists and media frame social problems: critical events versus performance trends for schools. Political communication, 12 (1), p. 5-26.

Sidicaro, R. (1998). Consideraciones a propósito de las ideas del diario La Nación. En: Wainterman, C. \& Sautu, R. (eds.). La trastienda de la investigación (p. 79-96). Buenos Aires: Lumiere. 
Ulanovsky, C. (1997). Paren las rotativas: historia de los grandes diarios, revistas y periodistas argentinos. Buenos Aires: Espasa Calpe.

Weaver, D.; McCombs, M. \& Shaw, D. L. (2004). Agenda-setting research: issues, attributes, and influences. En su: Handbook of political communication research (p. 257-282). New York: Lawrence Erlbaum Associates.

Wolf, M. (1987). La investigación en la comunicación de masas: crítica y perspectivas (3a. ed.). Buenos Aires: Paidós.

Wright, C. R. (1959). Mass communication: a sociological perspective (2nd ed.). New York: Random House.

Zallo, R. (1998). Economía de la comunicación y la cultura. Madrid: Akal.

Zhu, J. (1992). Issue competition and attention distraction: a zero-sum theory of agenda-setting. Journalism \& Mass Communication Quarterly, 69 (4), p. 825-836.

Zunino, E. (2013). La cobertura mediática de las elecciones presidenciales de 2011 en Argentina: un estudio sobre las agendas de los principales diarios del país. Temas de comunicación, (25), p. 90-104.

Zunino, E. (2015). La relevancia de las noticias en la prensa gráfica: una reflexión teórico metodológica a partir del análisis del conflicto entre las corporaciones agrarias y el gobierno argentino en 2008. Comunicación y sociedad (Guadalajara), 25 , p. 127-156. 